\title{
Molecular basis of inherited thrombocytopenias
}

\begin{tabular}{|r|l|}
\hline Journal: & Clinical Genetics \\
\hline Manuscript ID: & CGE-00050-2015.R2 \\
\hline Manuscript Type: & Review \\
\hline Date Submitted by the Author: & 04-May-2015 \\
\hline Complete List of Authors: & $\begin{array}{l}\text { Savoia, Anna; IRCCS Burlo Garofolo, Institute for Maternal and Child } \\
\text { Health; University of Trieste, Department of Medical Sciences }\end{array}$ \\
\hline Key Words: & $\begin{array}{l}\text { Inherited thrombocytopenia, Megakaryocytopoiesis, Molecular basis, } \\
\text { Platelet biogenesis }\end{array}$ \\
\hline
\end{tabular}

\section{SCHOLARONE}


Molecular basis of inherited thrombocytopenias

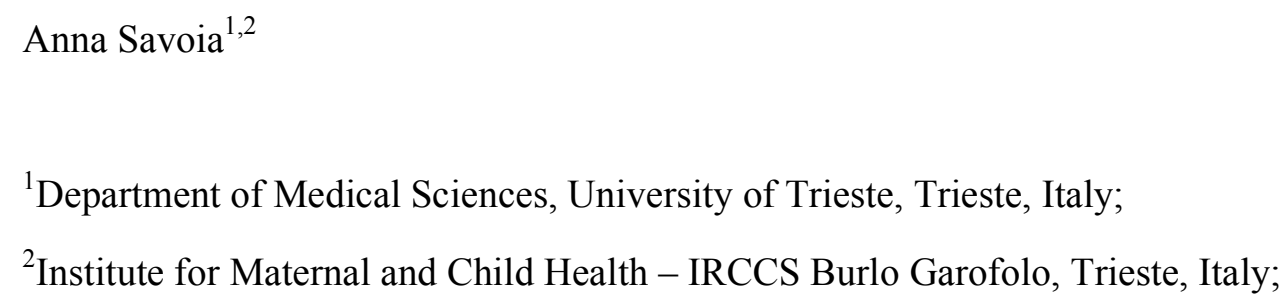

Corresponding author:

Dr. Anna Savoia

Department of Medical Sciences, University of Trieste

IRCCS Burlo Garofolo

Via dell'Istria 65/1 - 34137 Trieste, Italy

Tel +390403785527 - Fax +390403785540

Email: anna.savoia@burlo.trieste.it

\section{Acknowledgements}

This work was supported by grants GGP13082 from Telethon Foundation, Italian Ministry of Health (Ricerca Finalizzata 2010), and 15/12 and 16/12 of IRCCS Burlo Garofolo.

\section{Conflict of interest}

The author declares no conflict of interest 


\begin{abstract}
Inherited thrombocytopenias (IT) are a heterogeneous group of diseases caused by at least 26 different genes. At present, these genes account for approximately 50\% of cases, suggesting that novel genes have yet to be identified for a comprehensive understanding of platelet biogenesis defects. This review provides an update of ITs focusing on the molecular basis and potential pathogenic mechanisms affecting megakaryopoiesis and platelet production.
\end{abstract}

\title{
Key words
}

Inherited thrombocytopenia

Megakaryocytopoiesis

Molecular basis

Platelet biogenesis 


\section{Introduction}

Inherited thrombocytopenias (IT) are a heterogeneous group of diseases characterized by variable expressivity of the bleeding tendency due to low platelet count sometimes associated with platelet dysfunction. Platelets, whose normal count ranges between 150 and $450 \times 10^{9} / \mathrm{L}$, are quiescent when they circulate in the blood stream. After vessel injury they bind to the subendothelial von Willebrand factor, releasing the content of their granules, which draws other platelets to the site to prevent blood loss.

Thanks to the application of next generation sequencing, novel ITs have recently been described, improving our knowledge not only in definition of the clinical spectrum of ITs but also into the molecular mechanisms of platelet biogenesis. At present, mutations in more than 20 different genes are known to cause ITs. However, these genes account for approximately $50 \%$ of the IT patients, suggesting that novel forms are still to be characterized (1). The IT genes play a variety of roles in megakaryopoiesis and platelet production though their function is often unclear and needs to be further investigated. Although information is limited in many cases, this review aims at classifying ITs according to our knowledge of their molecular mechanisms. After a brief description of megakaryopoiesis and platelet release, the diseases are subdivided into three major groups, based on which phase of the cellular process leading to platelet production is defective (Fig. 1).

\section{Megakaryopoiesis and platelet production}

Platelets are generated from megakaryocytes (Mk) through a complex process that requires thrombopoietin (THPO) (Fig. 1). The binding of THPO to its receptor (MPL) results in dimerization of MPL and activation of a signaling cascade for commitment of hematopoietic stem cells to Mks and other blood cells (2). Although it is a continuous process, megakaryopoiesis could be distinguished into two phases, proliferation and maturation. During the early phase committed cells proliferate and differentiate into immature Mks. Then, the immature cells undergo endomitosis, a hallmark of the maturation process, accumulating DNA up to a content of $128 \mathrm{n}$. They produce large amounts of mRNA and proteins, including those for platelet adhesiveness, hemostasis and wound healing that are stored into $\alpha$-granules, the most abundant organelles in platelets (3).

Mature Mks migrate from the osteoblastic to the vascular niche of the bone marrow, where they form pseudopodia_(Fig. 1). Pseudopodia elongate and branch generating long processes (proplatelets) extending into the sinusoidal blood vessels (4). Proplatelet formation is mainly driven by apoptotic pathways and remodeling of the microtubule cytoskeleton $(5,6)$. Microtubules of $\alpha / \beta$-tubulin dimers organize into thick bundles where the pseudopodia originate and elongate forming increasingly narrower bundles as the proplatelets extend. At the distal end, the microtubule bundle loops and generates a teardrop-shaped structure that is released into the blood stream as a preplatelet, an intermediate form then converted into single platelets (6).

Proliferation and maturation of Mks, or proplatelet formation could be affected by disease-causing variants in genes controlling the different phases of platelet biogenesis_(Fig.1). When the early phases of megakaryopoiesis are defective, proliferation of the committed cells is impaired resulting in absence or reduction of 
Mks in the bone marrow. In case of defective maturation, Mks are normal or even increased in number but immature, being small and with a hypolobulated nucleus and/or reduced $\alpha$-granules. Thrombocytopenia associated with normal Mk production has been considered a hallmark of defective proplatelet formation, though platelet removal might also contribute to thrombocytopenia.

\section{Defects in the early phase of megakaryopoiesis: lack or reduction of Mk production}

Due to the key role of the THPO/MPL pathway, loss-of-function variants in the MPL gene cause congenital amegakaryocytic thrombocytopenia (CAMT; OMIM 604498). CAMT is a severe autosomal recessive disease with a high risk of life-threatening hemorrhages that evolves to bone marrow aplasia (7). Many different pathogenic variants of $M P L$ have been identified in homozygous or heterozygous compound subjects (7-9). Consistent with genotype/phenotype correlations, complete loss of the MPL function is associated with permanently low platelet counts, while in the case of residual activity there is transient improvement of thrombocytopenia within the first year of life (7). The $M P L$ variants explain approximately $60 \%$ of amegakaryocytic individuals, suggesting genetic heterogeneity of CAMT (7).

On the contrary, heterozygous gain-of-function variants of the THPO gene are associated with increased platelet production $(10,11)$. However, at least one loss-offunction variant (p.R38C), which is likely to reduce the binding affinity of THPO for MPL, has been identified in a large Micronesian family (12). Whereas the heterozygous individuals have mild thrombocytopenia, the homozygous ones develop aplastic anemia, supporting the role of the THPO/MPL pathway in the hematopoietic stem cell regulation. Similarly, microdeletions at 3q26.33-3q27.2 containing THPO are associated with mild thrombocytopenia $(13,14)$, suggesting that platelet production might be sensitive to THPO dosage.

There are at least another two ITs that could be included in this group, radioulnar synostosis with amegakaryocytic thrombocytopenia (RUSAT; OMIM 605432) and thrombocytopenia-absent radius syndrome (TAR; OMIM 274000), both associated with skeletal defects of the radius. Thrombocytopenia is usually severe due to absence or reduction of Mks in the bone marrow but, whereas it might evolve into aplastic anemia in RUSAT, the platelet count tends to increase during life in TAR individuals. RUSAT is a rare autosomal dominant IT that can be caused by variants of the HOXA11 gene (15). HOXA11 encodes for a homeobox protein that regulates gene expression, morphogenesis, and differentiation, whose role in hemopoiesis is not clear.

TAR was first associated with a microdeletion on chromosome 1q21.1 (16), which was, however, present in many healthy family members. Thanks to next generation strategies, one of the two low-frequency SNPs (rs139428292 in 5'UTR and rs201779890 in intron 1) of the hemizygous $R B M 8 A$ gene was identified in the affected individuals (17). Identification of compound heterozygotes carrying one null allele and one of the two SNPs on the other allele further supported the role of $R B M 8 A$ in TAR. Since $R B M 8 A$ is down regulated in TAR platelets, the two SNPs are likely to be hypomorphic variants affecting regulatory regions. $R B M 8 A$ encodes for RNA-binding protein $8 \mathrm{~A}$, a subunit of the exon-junction complex that is required for 
mRNA metabolism processes, such as splicing, mRNA export, and nonsensemediated mRNA decay. However, the role of RBM8A in megakaryopoieis remains unknown, though its low expression is likely to affect mRNA processing of components of the THPO/MPL pathway, as platelets of TAR individuals do not respond to recombinant THPO (18).

\section{Defective maturation: production of immature Mks}

Defects of megakaryopoiesis may also appear later, during the maturation of Mks. Interestingly, most of the ITs with defects in this phase are associated with alterations of genes encoding for transcription factors. These constitute a complex network of numerous elements, such as RUNX1, a master regulator of hematopoiesis, FLI1 regulating divergence of bipotential megakaryocytic/erythroid progenitors into platelets, and GATA1, FOG1, or GFI1B, which play a role in megakaryocytic and erythroid lineages.

Three of the ITs included in this group are characterized by an increased risk of developing hematological malignancies, including the familial platelet disorder with propensity to acute myelogenous leukemia (FPD-AML; OMIM 601399), which is caused by mutations in RUNX1. In addition to moderate thrombocytopenia, individuals with FPD-AML are at risk of developing myelodysplastic syndromes or acute myeloid leukemia (19). In PFD-AML, Mks are small but increased in number and platelets have a low content of $\alpha$-granules. RUNX1 interacts with subunit $\beta$ of the core-binding factor $(\mathrm{CBF}-\beta)$, which protects it from degradation and enhances its affinity for DNA (20). Different mutations have a haploinsufficiency effect, though a few missense variants act in a dominant-negative manner (21). Retaining the ability to interact with CBF- $\beta$ and/or the DNA binding activity, the dominant-negative products compete with the wild-type protein leading to a higher risk for hematological malignancies.

RUNX1 down-regulates the expression of ANKRD26, a gene associated with ANKRD26-related thrombocytopenia (ANKRD26-RT or THC2; OMIM 188000). In this autosomal dominant IT, Mks are increased in number with evidence of dysmegakaryopoiesis due to small Mks with hypolobulated nuclei and reduced $\alpha$ granule content (22). Moreover, Mks and platelets have particulate cytoplasmic structures (PaCS), consisting of accumulation of proteasome complexes and polyubiquitinated proteins that might contribute to thrombocytopenia (23). As in FPD-AML, patients are at risk of developing hematological malignancies, in particular acute myeloid leukemia $(22,24)$. Variants associated with the disease are all localized in a short stretch of nucleotides from c.-134G to c.-113A of the 5' UTR $(22,25,26)$. Since the molecular genetic testing of $A N K R D 26$ is usually directed only to the 5'UTR, it is likely that alterations of the coding region are underestimated. Indeed, one germline and one somatic amino acid substitution have been identified in a thrombocytopenic family and in an individual with acute myeloid leukemia, respectively $(27,28)$.

Data from in vitro assays suggests that the ANKRD26 variants, as the SNPs in $R B M 8 A$, alter the mechanisms controlling gene expression (25). Indeed, RUNX1 binds the 5'UTR stretch inhibiting the ANKRD26 transcription progressively along the Mk differentiation up to almost the complete absence of mRNA in mature cells (26). Instead, when the 5' UTR is mutated, the expression of ANKRD26 persists, 
resulting in constitutive activation of the TPO/MPL signaling, mainly of the MAPK/ERK1/2 pathway. Since the inhibition of this pathway is fundamental for proplatelet formation, patients' Mks have profound defects in proplatelet formation (23).

ETV6 is another tumor suppressor, whose somatic alterations have been associated with leukemia or myelodysplastic syndromes (29). It is a transcription factor of the ETS family characterized - as the other members - by the pointed (PNT) domain, which is involved in dimerization and interaction with other factors such as FLI1, a central region regulating the inhibitory effect, and the DNA-binding domain (ETS) (30). Four different germline missense variants affecting the central or the ETS domain have recently been identified in families with thrombocytopenia and different hematological malignancies (ETV6-related thrombocytopenia; ETV6-RT) (31, 32). Consistent with a maturation defect, Mks are small and hypolobulated in ETV6-RT individuals. The mutations prevent ETV6 from migrating into the nucleus and transcriptionally repressing target genes. Although knockout mice are embryonic lethal, the role of ETV6 during hematopoiesis has been established in megakaryocytic/erythroid conditional animal models. They have thrombocytopenia and an increased number of immature Mks, implicating the importance of ETV6 in platelet biogenesis $(33,34)$.

Like ETV6, FLI1 is a member of the ETS family and cooperates with RUNX1 during megakaryopoiesis (35). FLII is localized at 11q24.3, a chromosomal region that is deleted in Jacobsen syndrome (JBS; OMIM 147791). In addition to several dysmorphic features due to hemizygosity of different genes, JBS individuals have thrombocytopenia (also known as Paris-Trousseau syndrome - TCPT; OMIM 188025) (36). In TCPT, Mks are small with low ploidy level and have major defects in proplatelet production; platelets are increased in size with giant $\alpha$-granules (37). The hypothesis that haploinsufficiency of FLI1 is responsible for thrombocytopenia is supported by observations that overexpression of FLII restores megakaryopoiesis in hemizygous hematopoietic staminal cells (30). Moreover, point alterations have recently been identified in a few families with excessive bleeding (38). In these cases, platelets are not only reduced in number but have also defective aggregation. Of note, like RUNX1, FLI1 binds to the 5' UTR region of ANKRD26 in a consensus sequence close to the mutated stretch and synergistically has an inhibitory effect on the gene transcription $(22,26)$.

GATA1 is another transcription factor that together with RUNX1 and FLI1 regulates megakaryopoiesis (39). It controls biogenesis of both erythrocytes and platelets, which originate from a common bipotential megakaryocytic/erythroid progenitor. Indeed, variants of GATA1 cause an X-linked thrombocytopenia with either dyserythropoietic anemia (XLTDA; OMIM 300367) or $\beta$-thalassemia (XLTT; OMIM 314050) (40). In these GATAl-related diseases (GATAl-RD), maturation of Mks is severely compromised and platelets are large with reduced $\alpha$-granule content. GATA1 consists of one N-terminal (Nf) and one C-terminal (Cf) zinc finger domain. The different GATA1 variants affect the Nf domain, which interacts with FOG1 (friend of GATA1) with one surface and binds to DNA with the opposite surface. Amino acid substitutions p.V205M, p.G208R/S, and p.D218G/Y impair the GATA1/FOG1 interaction and are associated with dyserythropoietic anemia. Affecting the DNA binding surface, the sixth p.R216Q substitution is typical of GATA1-RD individuals with $\beta$-thalassemia (41). However, the GATA1 variants do not 
explain completely the patients' phenotype (42), suggesting that further investigations are needed to better understand the pathophysiological mechanisms underlying the hematological features.

Another transcription factor governing the maturation of Mks and erythroid cells is the GFI1B repressor. Two null variants affecting the DNA binding domain prevent repression of target genes $(43,44)$. They exert a dominant negative effect when coexpressed with the wild type form, consistent with the Gfilb+/- mouse, which has no blood abnormalities (45). Individuals carrying the GFIIB mutations have dysmorphic Mks, moderate thrombocytopenia with large platelets showing variable reduction, including absence in some cells, of the $\alpha$-granule content, and subtle red cell phenotype characterized by anisopoikilocytosis $(43,44)$. The phenotype of the GFIIB-related thrombocytopenia (GFIIB-RT; OMIM 187900) is similar to that of individuals with GATA1-RD, suggesting that GFI1B and GATA1 cooperate in the same cascade of events controlling the expression of genes essential for platelet and red cell formation. As for the other mutated transcription factors, identification of the target genes would provide relevant insight into the molecular pathways that are important for megakaryopoiesis and platelet production.

As mentioned, platelet $\alpha$-granules are variably reduced in platelets from different ITs, including ANKRD26-RT, GATA1-RD, and GFIIB-RT (43). On the contrary, platelets are typically gray in gray platelet syndrome (GPS; OMIM 139090) due to the complete lack of $\alpha$-granules. In addition to thrombocytopenia with large platelets, GPS individuals have additional features, such as splenomegaly, myelofibrosis and increased serum B12. Although GPS was a well-known disease, the gene (NBEAL2) was identified only thanks to next generation sequencing approaches (46-48). The absence of the $\alpha$-granules is observed only in individuals with biallelic variants of $N B E A L 2$, as none of the patients with mild $\alpha$-granule defect had pathogenic alterations of the gene (49).

NBEAL2 localizes to the dense tubular system and endoplasmic reticulum, where it is likely to function in vesicle trafficking and membrane dynamics (47). However, why loss-of-function variants cause GPS is unclear, though animal models have been elucidating some aspects. Nbeal2-/- knockout mice recapitulate the characteristics of GPS with platelet defects, splenomegaly and myelofibrosis (50-52). Whereas Nbeal2 is required for sorting and packing molecules into $\alpha$-granules, it is not essential for generating the $\alpha$-granule membranes. It also plays a role in megakaryopoiesis, as maturation of the Nbeal2-/- Mks is delayed with decreased ploidy and survival (51). These cells produce mature $\alpha$-granules, suggesting that lack of Neabl2 does not impair $\alpha$-granule formation. However, they do not retain the organelles (52). Indeed, $\alpha$-granules release proteins that participate in hemostasis and thrombus formation, as well as in wound healing and inflammation (50). A link between Nebeal2 deficiency and myelofibrosis derives from finding that Nbeal2-/- Mks have a strong proinflammatory transcriptome signature (52). Therefore, the Nbeal2-/- mouse is a promising model to dissect not only the function of the gene in $\alpha$-granules but also the biological role of $\alpha$-granules.

\section{Defective proplatelet formation and platelet release}


The largest group of ITs is not associated with immature MKs but with defects of proplatelet formation and platelet release. This process involves dynamic reorganization of the cytoskeleton, signaling pathways, and apoptosis, aspects that are defective in various forms of ITs. In addition to impaired formation of proplatelets, thrombocytopenia might be related to increased clearance of platelets from circulation as it occurs in Wiskott-Aldrich syndrome.

\section{Defective cytoskeleton components}

MYH9-related disease (MYH9-RD) is an autosomal dominant disorder caused by variants of $M Y H 9$, the gene encoding for the heavy chain of non-muscle myosin IIA (NMMHC-IIA) (53). NMMHC-IIA is expressed in most non-muscle cells, where it participates in several processes requiring chemomechanical forces. It consists of distinct domains, at the N-terminal a globular head domain followed by a coiled coil domain, and a non-helical tail at the C-terminus. Like the other conventional myosins, NMMHC-IIA dimerizes and associates with two pairs of regulatory and essential light chains. $M Y H 9-\mathrm{RD}$ is characterized by thrombocytopenia with giant platelets and typical inclusions in the cytoplasm of granulocytes. The majority of MYH9-RD patients develop, as late onset, extra-hematological manifestations, sensorineural hearing loss, presenile cataract, proteinuric nephropathy, and chronic or intermittent elevation of liver enzymes (54).

Of note is the spectrum of the MYH9 variants, mainly consisting of amino acid substitutions at a few of the NMMHC-IIA residues (53). Others are in-frame deletions or duplications in exon 24 or frame-shift and nonsense variants in the non-helical tail (exon 40). Variants at residues S96 and R702 in the globular head, R1165, D1424, and E1841 in the coiled coil domain, and R1933 in the non-helical tail account for almost $80 \%$ of the affected individuals. Considering that variants are detected in different populations and are often de novo events ( $>30 \%)$, it is plausible that only specific alterations of $M Y H 9$ are responsible for the disease.

Genotype-phenotype studies demonstrate strong correlations. In general, alterations of the head domain are associated with the most severe phenotype characterized by low platelet number and early onset of extra-hematological features, whereas variants of the tail domain segregate with low risk for extra-hematological features (55). When these studies were extended to large cases series, the correlations were supported even with single variants, such as those affecting residue R702 that are strongly associated with early-onset end-stage renal disease and deafness or p.D1424H, whose carriers are at high risk of developing all the manifestations (56). By contrast, patients with p.E1841K, p.D1424N or C-terminal null variants have no or very low risk. All the MYH9 variants cause MYH9-RD, though with variable expressivity. This is true even for $\mathrm{p} . \mathrm{R} 705 \mathrm{H}$, an amino acid substitution that was specifically associated with an autosomal dominant non-syndromic form of sensorineural hearing loss DFNA17. Only recently has it been reported that the p.R705H individuals have not only deafness but also the hematological defects and elevation of liver enzymes typical of MYH9-RD (57).

Considering the pathophysiological aspects, the Myh9+/- mice do not have any alteration whereas the Myh9-/- double mutants are lethal early during embryogenesis (58, 59). As human MYH9-RD, conditional animals have thrombocytopenia, large 
platelets, and an increased bleeding time (60). Reproducing the same molecular conditions as in patients, mice heterozygous for most frequent variants (p.R702C, p.D1424N, and p.E1841K) recapitulate the hematological, eye, and kidney manifestations (61). Not only mouse and human MYH9 mutants but also pharmacological inhibition of NMMHC-IIA increase proplatelet formation (62), an apparent paradox considering that variants are associated with thrombocytopenia. However, the MYH9-RD defect is likely to rely on lack of NMMHC-IIA reactivation in terminal platelet processing. Proplatelets are projected into microcirculation where shear facilitates proplatelet detachment. Fluid stress activates NMMHC-IIA, which accumulates at the stressed sites to induce fragmentation of preplatelets into normal sized platelets. If inhibited or mutated, NMMHC-IIA is diffuse and does not allow proplatelets to divide into small platelets, thus leading to large platelets in reduced number (63).

Myosins interact with actin filaments generating contractile forces. The long actin filaments (F-actin) are constituted of globular actin monomers (G-actin) that dynamically attach and detach at the ends of the filaments. The actin polymerization and depolymerization processes are promoted by nucleation factors, such as the Wiskott-Aldrich syndrome protein (WASp), which is selectively expressed in hematopoietic cells (64). It exists in a close auto-inhibited form characterized by interaction of the C-terminus (VCA domain) with other central domains, a structure that is stabilized by the WASP-interacting protein (WIP) binding to the N-terminus. Upon activation, WASp assumes an open conformation; the VCA domain binds a Gactin monomer, which is transferred to the Arp $2 / 3$ complex and added to F-actin. Therefore, alterations of the VCA domain result in complete loss of the WASpmediated actin polymerization.

Variants of the WAS gene are responsible for WAS (OMIM 301000) and X-linked thrombocytopenia (XLT; OMIM 313900), as well as a rare form of neutropenia with associated myelodysplastic syndromes $(65,66)$. In the WAS and XLT diseases, males have moderate to severe thrombocytopenia with small platelets. The WAS individuals also have a severe immune dysregulation with susceptibility to infections and eczema (67-69). A wide spectrum of different WAS alleles have been described with strong correlations between genotype and phenotype. Loss-of-function variants, such as nonsense and frameshift disrupting the VCA domain, are associated with the severe WAS phenotype. There are, instead, amino acid substitutions mainly affecting the WIP interacting domain that allow expression, even at reduced levels, of normal-sized hypomorphic WASp and are associated with XLT. Consistent with mouse models (70), thrombocytopenia is due not only to inefficient platelet release into the blood stream but also to increased clearance of platelets from circulation. Although patients' Mks are normal in number and proplatelet extension, they release platelets prematurely in the bone marrow. Moreover, morphologically defective platelets are likely to be phagocytized by the bone marrow or spleen macrophages, as splenectomy improves the count and quality of patients' platelets. For mechanisms explaining the other phenotypic aspects of this relatively well-known IT, the interested reader is referred to the literature (64).

Actin filaments are linked to $\alpha$-actinin, a cytoskeleton protein organized in an actinbinding domain (ABD), four spectrin repeats and a calmodulin-like domain (CaM). Antiparallel molecules dimerize in rod-like structures with $\mathrm{ABD}$ at each extremity for cross-linking the actin filaments into bundles. Among the four known isoforms, 
ACTN1 and, to a lesser extent, ACTN4 are expressed in Mks and platelets. Variants of ACTN1 cause the ACTN1-related thrombocytopenia (ACTN1-RT; OMIM 615193), a mild form of IT without any apparent associated features $(71,72)$. It is worth noting that all the 13 different pathogenic variants identified so far are amino acid substitutions residing within the $\mathrm{N}$ - and $\mathrm{C}$-terminal functional domains. Exogenous expression of the mutant proteins prevents actin from assembling into filaments, suggesting a dominant negative effect of the ACTN1 variants. Moreover, patients' Mks extend proplatelets at a normal rate but their tips are reduced in number and increased in size, suggesting a defect in the late phase of proplatelet formation (71).

Other actin-binding proteins are filamins, dimers connecting the actin filaments to the cellular membrane. In platelets, the most abundant isoform is filamin $A$, which is encoded by FLNA, a gene located on chromosome X. It has an ABD at the Nterminus, 24 Ig-like repeats, and a dimerization domain at the C-terminus. Variants of FLNA determine a wide spectrum of rare brain, heart and muscle developmental diseases, including periventricular nodular heterotopia. The periventricular nodular heterotopia has sometimes been associated with thrombocytopenia, as reported in three females heterozygous for loss-of-function variants of FLNA (73). Consistent with a role of FLNA in platelet biogenesis, a novel missense variant was identified in an isolated thrombocytopenia (74). Moreover, a recent study showed that individuals with FLNA variants have defective platelet aggregation and secretion (75). Together with macrothrombocytopenia, similar alterations were observed in conditional mice, further supporting the role of FLNA in platelet production and function (76).

FLNA, as well GPIb $\beta$ (see below), is one of the cAMP-dependent protein kinase A substrates. This kinase, which consists of two regulatory and two catalytic subunits, phosphorylates FLNA at S2152 protecting it from proteolysis (77). The gene encoding for the $\gamma$-isoform of the catalytic subunit, $P R K A C G$, is mutated in an autosomal recessive form of macrothrombocytopenia. The only known variant (p.I74M) has been identified in one consanguineous family without any other features associated except for platelet dysfunction due to defective aggregation (78). In patients' Mks and platelets, whereas the GPIb $\beta$ phosphorylation status is in the normal range, FLNA is expressed at low levels, suggesting a rapid degradation of the protein. Moreover, the percentage of proplatelet-bearing Mks is significantly reduced in affected individuals, a defect that can be complemented by overexpression of the wild-type gene, further supporting the role of PRKACG in platelet biogenesis (78).

Other principal components of the cytoskeleton are microtubules, dynamic structures that, by undergoing continuous assembly and disassembly, drive a variety of cellular functions, such as proplatelet formation and elongation. They are heterodimers of $\alpha$ and $\beta$-tubulin subunits. TUBB1, the gene encoding for the $\beta 1$ isoform, is responsible for the TUBB1-related thrombocytopenia (TUBB1-RT; OMIM 613112) (79, 80). Since the two $T U B B 1$ variants identified so far are located at or near the intradimer interface, it is likely that the mutant forms interfere with the microtubule assembly. $T U B B 1$ is exclusively expressed in Mks and platelets, suggesting that TUBB1 functions in late stages of megakaryopoiesis (81). Consistent with data on patients' peripheral blood derived-Mks, overexpression of mutant forms do not allow microtubules to assemble and cells generate few proplatelets with large tips. Similar defects have been observed in mouse and dog models $(82,83)$, further supporting the hypothesis that TUBB1-RT is due to microtubule disorganization. 
As mentioned, FLNA anchors the actin filaments to membrane glycoproteins, providing potential transduction of signals to the cytoskeleton network. Indeed, FLNA interacts with the cytoplasmic domain of GPIb $\alpha$, one of the subunits of the receptor (GPIb-IX-V) for the von Willebrand factor (VWF) (84). In addition to GPIba, the GPIb-IX-V complex consists of another three subunits, GPIb $\beta$, GPIX and GPV, that associate in the ratio $2: 4: 2: 1$ in the endoplasmic reticulum before localizing at the cell surface $(85,86)$. Variants of the $G P 1 B A, G P 1 B B$, and $G P 9$ genes encoding for the GPIb $\alpha$, GPIb $\beta$, and GPIX subunits, respectively, cause Bernard-Soulier syndrome (BSS; OMIM 231200). There is a wide spectrum of variants that prevent the complex from associating and migrating to the platelet membrane or more rarely from interacting with VWF (87). As a consequence, platelets are unable to adhere to the vascular subendothelium and aggregate, leading to severe bleeding tendency. In addition to functional defects, BSS platelets are reduced in number and bigger than red cells.

Although BSS is an autosomal recessive disease, monoallelic variants of GP1BA and $G P 1 B B$ are associated with mild macrothrombocytopenia transmitted as a dominant trait (OMIN 153670) (87). At least in Italy, there is a significant contribution of p.A172V because of a founder effect (88). For a comprehensive description of ITs, it is worth mentioning platelet-type von Willebrand disease, a macrothrombocytopenia due to GPIBA variants that increase the affinity of GPIb $\alpha$ for VWF (87). Paradoxically, both a lack and excess of GPIb $\alpha$ stimulation produce the same effects on platelet biogenesis. Therefore, the interaction between VWF and GPIb $\alpha$ has to be finely modulated to guarantee the activation of specific signaling pathways through the interaction of the GPIb-IX-V receptor with the cytoskeleton network. Consistent with this hypothesis, proplatelet formation is significantly reduced from Mks of BSS individuals (89), though further studies are required to unravel the origin of thrombocytopenia.

Another important complex of hemostasis is GPIIb-IIIa. When active, it exposes the binding site for fibrinogen, resulting in platelet aggregation. Biallelic variants of $I T G A 2 B$ and ITGB3, the genes encoding for the GPIIb and GPIIIa integrin subunits of the receptor, cause Glanzmann thrombasthenia, an autosomal recessive bleeding disorder with normal platelet count. There are, however, gain-of-function variants affecting the GPIIb and GPIIIa regions proximal to the membrane that lead to a constitutive activation of the receptor (90-94). Individuals with these variants have an autosomal dominant macrothrombocytopenia without aggregation defects, suggesting that the GPIIb-IIIa receptor has to properly interact with the fibrinogen of the extracellular matrix of the bone marrow for platelet biogenesis $(91,95)$.

\section{Defects of apoptosis}

The intrinsic apoptotic pathway residing on release of cytochrome c (CYCS) from mitochondria is essential for proplatelet formation (5). Indeed, two variants (p.G42S and p.Y49H) of the CYCS gene are associated with CYCS-related thrombocytopenia (CYCS-RT or THC4; OMIM 612004) $(96,97)$. In vitro studies support the hypothesis that the mutant proteins determine reduction of respiration and higher apoptotic rate, favoring at least for the p.G42S mutant the interaction with apoptotic protease- 
activating factor 1 and consequent caspase activation (96). Dysregulation of megakaryopoiesis is consistent with naked Mk nuclei and platelets observed in the bone marrow and premature in vitro production of platelets from hematopoietic stem cells. This data supports the hypothesis that the intrinsic apoptotic signaling pathway is finely modulated during the platelet formation process.

\section{Conclusions}

ITs are relatively rare diseases not always recognized as inherited forms leading to misdiagnosis of affected individuals as having immune thrombocytopenia. Considering that clinical and hematological features are often similar among the different ITs and that almost $50 \%$ of families have yet uncharacterized forms of IT (1, 98), their diagnosis is a complex process requiring a multistep approach. The review is limited to the genetic and pathophysiological aspects of ITs and, for a more comprehensive review of clinical and diagnostic aspects, the interested reader is referred to a paper by Pecci (2015) published in Clinical Genetics. 
Figure legend

Fig. 1. Schematic representation of megakaryopoiesis and platelet release. The cartoon represents the different phases of plateletet biogenesis, including differentiation and maturation of megakaryocytes, proplatelet production and platelet release into blood stream. In ITs, each phase could be affected by variants of genes that are also indicated.

References

1. Balduini CL, Savoia A. Genetics of familial forms of thrombocytopenia. Human Genetics 2012: 131: 1821-1832.

2. Olson TS, Caselli A, Otsuru S et al. Megakaryocytes promote murine osteoblastic HSC niche expansion and stem cell engraftment after radioablative conditioning. Blood 2013: 121: 5238-5249.

3. Machlus KR, Thon JN, Italiano JE. Interpreting the developmental dance of the megakaryocyte: a review of the cellular and molecular processes mediating platelet formation. Br J Haematol 2014: 165: 227-236.

4. Junt $\mathrm{T}$, Schulze $\mathrm{H}$, Chen $\mathrm{Z}$ et al. Dynamic visualization of thrombopoiesis within bone marrow. Science 2007: 317: 1767-1770.

5. Kile BT. The role of apoptosis in megakaryocytes and platelets. Br J Haematol 2014: 165: 217-226.

6. Thon JN, Italiano JE. Platelets: production, morphology and ultrastructure. Handb Exp Pharmacol 2012: 3-22.

7. Ballmaier M, Germeshausen M. Congenital amegakaryocytic thrombocytopenia: clinical presentation, diagnosis, and treatment. Semin Thromb Hemost 2011: 37: 673-681.

8. Ihara K, Ishii E, Eguchi $\mathrm{M}$ et al. Identification of mutations in the c-mpl gene in congenital amegakaryocytic thrombocytopenia. Proc Natl Acad Sci U S A 1999: 96: 3132-3136.

9. Savoia A, Dufour C, Locatelli F et al. Congenital amegakavyocytic thrombocytopenia: clinical and biological consequences of five novel mutations. Haematologica-the Hematology Journal 2007: 92: 1186-1193.

10. Wiestner A, Schlemper RJ, van der Maas AP et al. An activating splice donor mutation in the thrombopoietin gene causes hereditary thrombocythaemia. Nat Genet 1998: 18: 49-52.

11. Stockklausner C, Echner N, Klotter AC et al. Hereditary thrombocythemia caused by a thrombopoietin (THPO) gain-of-function mutation associated with multiple myeloma and congenital limb defects. Ann Hematol 2012: 91: 1129-1133.

12. Dasouki MJ, Rafi SK, Olm-Shipman AJ et al. Exome sequencing reveals a thrombopoietin ligand mutation in a Micronesian family with autosomal recessive aplastic anemia. Blood 2013: 122: 3440-3449.

13. Mandrile G, Dubois A, Hoffman JD et al. 3q26.33-3q27.2 microdeletion: a new microdeletion syndrome? Eur J Med Genet 2013: 56: 216-221. 
14. Dasouki M, Roberts J, Santiago A et al. Confirmation and further delineation of the 3q26.33-3q27.2 microdeletion syndrome. Eur J Med Genet 2014: 57: 76-80.

15. Thompson AA, Nguyen LT. Amegakaryocytic thrombocytopenia and radioulnar synostosis are associated with HOXA11 mutation. Nat Genet 2000: 26: $397-$ 398.

16. Klopocki E, Schulze H, Strauss G et al. Complex inheritance pattern resembling autosomal recessive inheritance involving a microdeletion in thrombocytopenia-absent radius syndrome. Am J Hum Genet 2007: 80: 232-240.

17. Albers CA, Paul DS, Schulze $\mathrm{H}$ et al. Compound inheritance of a lowfrequency regulatory SNP and a rare null mutation in exon-junction complex subunit RBM8A causes TAR syndrome. Nat Genet 2012: 44: 435-439, S431-432.

18. Ballmaier M, Schulze H, Strauss G et al. Thrombopoietin in patients with congenital thrombocytopenia and absent radii: elevated serum levels, normal receptor expression, but defective reactivity to thrombopoietin. Blood 1997: 90: 612-619.

19. Liew E, Owen C. Familial myelodysplastic syndromes: a review of the literature. Haematologica 2011: 96: 1536-1542.

20. Song WJ, Sullivan MG, Legare RD et al. Haploinsufficiency of CBFA2 causes familial thrombocytopenia with propensity to develop acute myelogenous leukaemia. Nat Genet 1999: 23: 166-175.

21. Matheny CJ, Speck ME, Cushing PR et al. Disease mutations in RUNX1 and RUNX2 create nonfunctional, dominant-negative, or hypomorphic alleles. EMBO J 2007: 26: 1163-1175.

22. Noris P, Perrotta S, Seri M et al. Mutations in ANKRD26 are responsible for a frequent form of inherited thrombocytopenia: analysis of 78 patients from 21 families. Blood 2011: 117: 6673-6680.

23. Necchi V, Balduini A, Noris $\mathrm{P}$ et al. Ubiquitin/proteasome-rich particulate cytoplasmic structures (PaCSs) in the platelets and megakaryocytes of ANKRD26related thrombo-cytopenia. Thromb Haemost 2013: 109: 263-271.

24. Noris P, Favier R, Alessi MC et al. ANKRD26-related thrombocytopenia and myeloid malignancies. Blood 2013: 122: 1987-1989.

25. Pippucci T, Savoia A, Perrotta S et al. Mutations in the 5 ' UTR of ANKRD26, the Ankirin Repeat Domain 26 Gene, Cause an Autosomal-Dominant Form of Inherited Thrombocytopenia, THC2. American Journal of Human Genetics 2011: 88: 115-120.

26. Bluteau D, Balduini A, Balayn $\mathrm{N}$ et al. Thrombocytopenia-associated mutations in the ANKRD26 regulatory region induce MAPK hyperactivation. J Clin Invest 2014: 124: 580-591.

27. Mardis ER, Ding L, Dooling DJ et al. Recurring mutations found by sequencing an acute myeloid leukemia genome. N Engl J Med 2009: 361: 1058-1066.

28. Al Daama SA, Housawi YH, Dridi W et al. A missense mutation in ANKRD26 segregates with thrombocytopenia. Blood 2013: 122: 461-462.

29. De Braekeleer E, Douet-Guilbert N, Morel F et al. ETV6 fusion genes in hematological malignancies: a review. Leuk Res 2012: 36: 945-961.

30. Raslova H, Komura E, Le Couédic JP et al. FLI1 monoallelic expression combined with its hemizygous loss underlies Paris-Trousseau/Jacobsen thrombopenia. J Clin Invest 2004: 114: 77-84.

31. Zhang MY, Churpek JE, Keel SB et al. Germline ETV6 mutations in familial thrombocytopenia and hematologic malignancy. Nat Genet 2015. 
32. Noetzli L, Lo RW, Lee-Sherick AB et al. Germline mutations in ETV6 are associated with thrombocytopenia, red cell macrocytosis and predisposition to lymphoblastic leukemia. Nat Genet 2015.

33. Wang LC, Swat W, Fujiwara Y et al. The TEL/ETV6 gene is required specifically for hematopoiesis in the bone marrow. Genes Dev 1998: 12: 2392-2402.

34. Hock H, Meade E, Medeiros S et al. Tel/Etv6 is an essential and selective regulator of adult hematopoietic stem cell survival. Genes Dev 2004: 18: 2336-2341.

35. Huang $\mathrm{H}, \mathrm{Yu} \mathrm{M}$, Akie $\mathrm{TE}$ et al. Differentiation-dependent interactions between RUNX-1 and FLI-1 during megakaryocyte development. Mol Cell Biol 2009: 29: 4103-4115.

36. Grossfeld PD, Mattina $\mathrm{T}$, Lai $\mathrm{Z}$ et al. The 11q terminal deletion disorder: a prospective study of 110 cases. Am J Med Genet A 2004: 129A: 51-61.

37. Bluteau D, Glembotsky AC, Raimbault A et al. Dysmegakaryopoiesis of FPD/AML pedigrees with constitutional RUNX1 mutations is linked to myosin II deregulated expression. Blood 2012: 120: 2708-2718.

38. Stockley J, Morgan NV, Bem D et al. Enrichment of FLI1 and RUNX1 mutations in families with excessive bleeding and platelet dense granule secretion defects. Blood 2013: 122: 4090-4093.

39. Tijssen MR, Cvejic A, Joshi A et al. Genome-wide analysis of simultaneous GATA1/2, RUNX1, FLI1, and SCL binding in megakaryocytes identifies hematopoietic regulators. Dev Cell 2011: 20: 597-609.

40. Millikan PD, Balamohan SM, Raskind WH et al. Inherited thrombocytopenia due to GATA-1 mutations. Semin Thromb Hemost 2011: 37: 682-689.

41. Balduini CL, Pecci A, Loffredo G et al. Effects of the R216Q mutation of GATA-1 on erythropoiesis and megakaryocytopoiesis. Thromb Haemost 2004: 91: 129-140.

42. Campbell AE, Wilkinson-White L, Mackay JP et al. Analysis of diseasecausing GATA1 mutations in murine gene complementation systems. Blood 2013: 121: 5218-5227.

43. Monteferrario D, Bolar NA, Marneth AE et al. A dominant-negative GFI1B mutation in the gray platelet syndrome. N Engl J Med 2014: 370: 245-253.

44. Stevenson WS, Morel-Kopp MC, Chen Q et al. GFI1B mutation causes a bleeding disorder with abnormal platelet function. J Thromb Haemost 2013: 11: 2039-2047.

45. Vassen L, Okayama T, Möröy T. Gfilb:green fluorescent protein knock-in mice reveal a dynamic expression pattern of Gfilb during hematopoiesis that is largely complementary to Gfi1. Blood 2007: 109: 2356-2364.

46. Albers CA, Cvejic A, Favier R et al. Exome sequencing identifies NBEAL2 as the causative gene for gray platelet syndrome. Nat Genet 2011: 43: 735-737.

47. Gunay-Aygun M, Falik-Zaccai TC, Vilboux T et al. NBEAL2 is mutated in gray platelet syndrome and is required for biogenesis of platelet $\alpha$-granules. Nat Genet 2011: 43: 732-734.

48. Kahr WH, Hinckley J, Li L et al. Mutations in NBEAL2, encoding a BEACH protein, cause gray platelet syndrome. Nat Genet 2011: 43: 738-740.

49. Bottega R, Pecci A, De Candia E et al. Correlation between platelet phenotype and NBEAL2 genotype in patients with congenital thrombocytopenia and alphagranule deficiency. Haematologica 2013: 98: 868-874.

50. Deppermann C, Cherpokova D, Nurden P et al. Gray platelet syndrome and defective thrombo-inflammation in Nbeal2-deficient mice. J Clin Invest 2013. 
51. Kahr WH, Lo RW, Li L et al. Abnormal megakaryocyte development and platelet function in Nbeal2(-/-) mice. Blood 2013: 122: 3349-3358.

52. Guerrero JA, Bennett C, van der Weyden L et al. Gray platelet syndrome: proinflammatory megakaryocytes and $\alpha$-granule loss cause myelofibrosis and confer metastasis resistance in mice. Blood 2014: 124: 3624-3635.

53. Balduini CL, Pecci A, Savoia A. Recent advances in the understanding and management of MYH9-related inherited thrombocytopenias. British Journal of Haematology 2011: 154: 161-174.

54. Pecci A, Biino G, Fierro T et al. Alteration of Liver Enzymes Is a Feature of the Myh9-Related Disease Syndrome. Plos One 2012: 7.

55. Pecci A, Panza E, Pujol-Moix N et al. Position of nonmuscle myosin heavy chain IIA (NMMHC-IIA) mutations predicts the natural history of MYH9-related disease. Human Mutation 2008: 29: 409-417.

56. Pecci A, Klersy C, Gresele P et al. MYH9-Related Disease: A Novel Prognostic Model to Predict the Clinical Evolution of the Disease Based on Genotype-Phenotype Correlations. Human Mutation 2014: 35: 236-247.

57. Verver E, Pecci A, De Rocco D et al. R705H mutation of MYH9 is associated with MYH9-related disease and not only with non-syndromic deafness DFNA17. Clin Genet 2014.

58. Conti MA, Even-Ram S, Liu $\mathrm{C}$ et al. Defects in cell adhesion and the visceral endoderm following ablation of nonmuscle myosin heavy chain II-A in mice. J Biol Chem 2004: 279: 41263-41266.

59. Matsushita T, Hayashi H, Kunishima S et al. Targeted disruption of mouse ortholog of the human MYH9 responsible for macrothrombocytopenia with different organ involvement: hematological, nephrological, and otological studies of heterozygous KO mice. Biochem Biophys Res Commun 2004: 325: 1163-1171.

60. Léon C, Eckly A, Hechler B et al. Megakaryocyte-restricted MYH9 inactivation dramatically affects hemostasis while preserving platelet aggregation and secretion. Blood 2007: 110: 3183-3191.

61. Zhang Y, Conti MA, Malide D et al. Mouse models of MYH9-related disease: mutations in nonmuscle myosin II-A. Blood 2012: 119: 238-250.

62. Chen Z, Naveiras O, Balduini A et al. The May-Hegglin anomaly gene MYH9 is a negative regulator of platelet biogenesis modulated by the Rho-ROCK pathway. Blood 2007: 110: 171-179.

63. Spinler KR, Shin JW, Lambert MP et al. Myosin-II repression favors pre/proplatelets but shear activation generates platelets and fails in macrothrombocytopenia. Blood 2015: 125: 525-533.

64. Massaad MJ, Ramesh N, Geha RS. Wiskott-Aldrich syndrome: a comprehensive review. Ann N Y Acad Sci 2013: 1285: 26-43.

65. Zhu Q, Zhang M, Blaese RM et al. The Wiskott-Aldrich syndrome and Xlinked congenital thrombocytopenia are caused by mutations of the same gene. Blood 1995: 86: 3797-3804.

66. Devriendt K, Kim AS, Mathijs G et al. Constitutively activating mutation in WASP causes X-linked severe congenital neutropenia. Nat Genet 2001: 27: 313-317.

67. Notarangelo LD, Miao CH, Ochs HD. Wiskott-Aldrich syndrome. Curr Opin Hematol 2008: 15: 30-36.

68. Mahlaoui N, Pellier I, Mignot C et al. Characteristics and outcome of earlyonset, severe forms of Wiskott-Aldrich syndrome. Blood 2013: 121: 1510-1516. 
69. Albert MH, Bittner TC, Nonoyama S et al. X-linked thrombocytopenia (XLT) due to WAS mutations: clinical characteristics, long-term outcome, and treatment options. Blood 2010: 115: 3231-3238.

70. Sabri S, Foudi A, Boukour S et al. Deficiency in the Wiskott-Aldrich protein induces premature proplatelet formation and platelet production in the bone marrow compartment. Blood 2006: 108: 134-140.

71. Kunishima S, Okuno Y, Yoshida K et al. ACTN1 mutations cause congenital macrothrombocytopenia. Am J Hum Genet 2013: 92: 431-438.

72. Bottega R, Marconi C, Faleschini M et al. ACTN1-related thrombocytopenia: identification of novel families for phenotypic characterization. Blood 2015: 125: 869-872.

73. Parrini E, Ramazzotti A, Dobyns WB et al. Periventricular heterotopia: phenotypic heterogeneity and correlation with Filamin A mutations. Brain 2006: 129: 1892-1906.

74. Nurden P, Debili N, Coupry I et al. Thrombocytopenia resulting from mutations in filamin A can be expressed as an isolated syndrome. Blood 2011: 118: 5928-5937.

75. Berrou E, Adam F, Lebret $\mathrm{M}$ et al. Heterogeneity of platelet functional alterations in patients with filamin A mutations. Arterioscler Thromb Vasc Biol 2013: 33: e11-18.

76. Falet H, Pollitt AY, Begonja AJ et al. A novel interaction between FlnA and Syk regulates platelet ITAM-mediated receptor signaling and function. J Exp Med 2010: 207: 1967-1979.

77. Jay D, García EJ, Lara JE et al. Determination of a cAMP-dependent protein kinase phosphorylation site in the C-terminal region of human endothelial actinbinding protein. Arch Biochem Biophys 2000: 377: 80-84.

78. Manchev VT, Hilpert M, Berrou $\mathrm{E}$ et al. A new form of macrothrombocytopenia induced by a germ-line mutation in the PRKACG gene. Blood 2014: 124: 2554-2563.

79. Kunishima S, Nishimura S, Suzuki $\mathrm{H}$ et al. TUBB1 mutation disrupting microtubule assembly impairs proplatelet formation and results in congenital macrothrombocytopenia. Eur J Haematol 2014: 92: 276-282.

80. Kunishima S, Kobayashi R, Itoh TJ et al. Mutation of the beta1-tubulin gene associated with congenital macrothrombocytopenia affecting microtubule assembly. Blood 2009: 113: 458-461.

81. Lecine P, Italiano JE, Kim SW et al. Hematopoietic-specific beta 1 tubulin participates in a pathway of platelet biogenesis dependent on the transcription factor NF-E2. Blood 2000: 96: 1366-1373.

82. Davis B, Toivio-Kinnucan M, Schuller S et al. Mutation in beta1-tubulin correlates with macrothrombocytopenia in Cavalier King Charles Spaniels. J Vet Intern Med 2008: 22: 540-545.

83. Schwer HD, Lecine P, Tiwari S et al. A lineage-restricted and divergent betatubulin isoform is essential for the biogenesis, structure and function of blood platelets. Curr Biol 2001: 11: 579-586.

84. Nakamura F, Stossel TP, Hartwig JH. The filamins: organizers of cell structure and function. Cell Adh Migr 2011: 5: 160-169.

85. Luo SZ, Mo X, Afshar-Kharghan V et al. Glycoprotein Ibalpha forms disulfide bonds with 2 glycoprotein Ibbeta subunits in the resting platelet. Blood 2007: 109: 603-609. 
86. López JA, Andrews RK, Afshar-Kharghan V et al. Bernard-Soulier syndrome. Blood 1998: 91: 4397-4418.

87. Savoia A, Kunishima S, De Rocco D et al. Spectrum of the mutations in Bernard-Soulier syndrome. Hum Mutat 2014: 35: 1033-1045.

88. Noris P, Perrotta S, Bottega R et al. Clinical and laboratory features of 103 patients from 42 Italian families with inherited thrombocytopenia derived from the monoallelic Ala156Val mutation of GPIb alpha (Bolzano mutation). Haematologicathe Hematology Journal 2012: 97: 82-88.

89. Balduini A, Malara A, Pecci A et al. Proplatelet formation in heterozygous Bernard-Soulier syndrome type Bolzano. J Thromb Haemost 2009: 7: 478-484.

90. Gresele P, Falcinelli E, Giannini S et al. Dominant inheritance of a novel integrin beta(3) mutation associated with a hereditary macrothrombocytopenia and platelet dysfunction in two Italian families. Haematologica-the Hematology Journal 2009: 94: 663-669.

91. Ghevaert C, Salsmann A, Watkins NA et al. A nonsynonymous SNP in the ITGB3 gene disrupts the conserved membrane-proximal cytoplasmic salt bridge in the alphaIIbbeta 3 integrin and cosegregates dominantly with abnormal proplatelet formation and macrothrombocytopenia. Blood 2008: 111: 3407-3414.

92. Jayo A, Conde I, Lastres P et al. L718P mutation in the membrane-proximal cytoplasmic tail of beta 3 promotes abnormal alpha IIb beta 3 clustering and lipid microdomain coalescence, and associates with a thrombasthenia-like phenotype. Haematologica 2010: 95: 1158-1166.

93. Kunishima S, Kashiwagi H, Otsu M et al. Heterozygous ITGA2B R995W mutation inducing constitutive activation of the $\alpha \operatorname{IIb} \beta 3$ receptor affects proplatelet formation and causes congenital macrothrombocytopenia. Blood 2011: 117: 54795484.

94. Kashiwagi H, Kunishima S, Kiyomizu K et al. Demonstration of novel gainof-function mutations of $\alpha \mathrm{IIb} \beta 3$ : association with macrothrombocytopenia and glanzmann thrombasthenia-like phenotype. Mol Genet Genomic Med 2013: 1: 77-86. 95. Larson MK, Watson SP. Regulation of proplatelet formation and platelet release by integrin alpha IIb beta3. Blood 2006: 108: 1509-1514.

96. Morison IM, Cramer Bordé EM, Cheesman EJ et al. A mutation of human cytochrome $\mathrm{c}$ enhances the intrinsic apoptotic pathway but causes only thrombocytopenia. Nat Genet 2008: 40: 387-389.

97. De Rocco D, Cerqua C, Goffrini P et al. Mutations of cytochrome c identified in patients with thrombocytopenia THC4 affect both apoptosis and cellular bioenergetics. Biochim Biophys Acta 2014: 1842: 269-274.

98. Noris P, Pecci A, Di Bari F et al. Application of a diagnostic algorithm for inherited thrombocytopenias to 46 consecutive patients. Haematologica 2004: 89: 1219-1225. 
Figure 1

1 5 6 7 8 9 10

26 ematopoietic 28 stem cell 29

$$
\text { niche }
$$

\begin{abstract}
Megakaryocyte differentiation and maturation
\end{abstract}

Proplatelet production

MYH9, WAS, ACTN1 FLNA, TUBB1

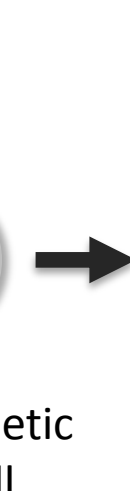

Immature megakaryocyte

\author{
RUNX1 \\ FLI1, ETV6 \\ GATA1, GFI1B \\ ANKRD26 \\ NBEAL2
}

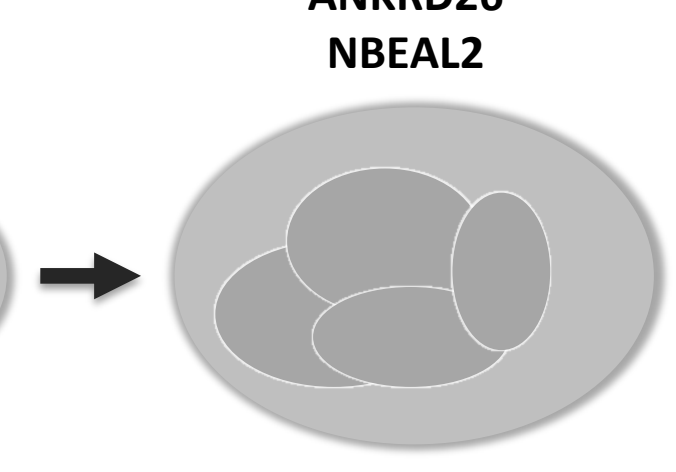

Mature

megakaryocyte
GP1BA,GP1BB, GP9

ITGA2B, ITGB3

CYCS

$$
\begin{aligned}
& \text { Vascular } \\
& \text { niche }
\end{aligned}
$$

Platelet release

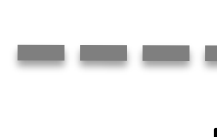

Megakaryocyte migration 